\title{
Acute Sensory Neuropathy and Progressive Dysphagia with Anti-GQ1b Antibody Positive: A Case Report
}

\author{
Sarawut Suksuphew ${ }^{* 1}$ and Chantira Chiaranai ${ }^{2}$ \\ ${ }^{1}$ School of Medicine (Neurology), Institute of Medicine, Suranaree University of Technology, Thailand \\ ${ }^{2}$ School of Adult and Elderly Nursing, Institute of Nursing,Suranaree University of Technology, Thailand
}

Received: 漹: December 31, 2018; Published: 制: January 09, 2019

*Corresponding author: Sarawut Suksuphew, School of Medicine (Neurology), Institute of Medicine, Suranaree University of Technology, Thailand

\begin{abstract}
Anti-GQ1b ganglioside antibody is associated with ophthalmoplegia, ataxia, and areflexia, resulting in the development of Miller Fisher Syndrome (MFS), Guillain-Barré Syndrome (GBS) with ophthalmoplegia and Bickerstaff brainstem encephalitis. We presented the case of a 43-yearold male with numbness of all extremities, progressive dysphagia and dysarthria. He had a history of flu-like symptoms before his numbness. Physical examination found decreased in pinprick sensation, limb ataxia, hyporeflexia and decreased response of gag reflex. The results of the MRI brain and cervical spine were found normal. The biochemical examination of cerebrospinal fluid was found normal. He was treated with Intravenous Immunoglobulin (IVIG) for five days. He has recovered completely and could return to work within four weeks. The result of his serum anti-GQ1b antibody was reported positive. Although clinical symptoms do not match the criteria diagnosis of MFS or GBS, however the evaluation of specific antibodies help in the diagnostics and thus early effective treatment is possible.
\end{abstract}

Keywords: Anti-GQ1b antibodies; Miller Fisher Syndrome; Guillain-Barre Syndrome

Abbreviations: MFS: Miller Fisher Syndrome; GBS: Guillain-Barré Syndrome; IVIG: Intravenous Immunoglobulin; MRI: Magnetic Resonance Imaging, MFS: Miller Fisher Syndrome, CSF: Cerebrospinal Fluid

\section{Introduction}

Anti-GQ1b antibody is associated with ophthalmoplegia, ataxia, and areflexia, resulting in the development of Guillain-Barré Syndrome (GBS) with ophthalmoplegia, Miller Fisher Syndrome (MFS), and Bickerstaff brainstem encephalitis which are collectively called "Anti-GQ1b Antibody Syndrome" [1-3].

\section{Case Report}

A 43-year-old male presented with a ten-day history of flulike symptoms. Five days after the onset of flu-like symptoms, he developed numbness in distal part of all extremities. Two days after this, he developed loss of balance. No impairment of consciousness, diplopia, dysphonia, dysphagia and motor weakness. On admission, the vital signs were found normal. The results from the pinprick test showed decreased of sensation in both sides, worse on the distal parts. Distal deep tendon reflexes were decreased. Limb strength with Grade V on the Medical Research Council scale and plantar responses were bilaterally flexor. Cerebellar tests were shown ataxic limbs. Routine blood tests including complete blood count, renal function test, glucose, electrolytes, calcium, magnesium, phosphorus were found normal. The radiological examination including cranial and cervical spine Magnetic Resonance Imaging (MRI) scans were found normal. Cerebrospinal Fluid (CSF) studies including cell count, biochemical and cytology were found normal; the CSF was clear colorless, with normal opening pressure. Electroneuro physiological examination was undergoing appointments. The most likely differential diagnosis was overlapping between GBS and MFS.

During his admission in ward, major changes in his symptoms occurred other than the limb numbness and impaired balance becoming progressive. He experienced dysarthria, difficulty swallowing saliva and difficulty breathing when lying on his back. Neurological examination in the state of progressive condition at that time found that he had a weakness in the bilateral facial muscles without ophthalmoplegia. The gag reflex was decreased bilaterally. Intravenous Immunoglobulin (IVIG) therapy $(0.4 \mathrm{~g} / \mathrm{kg} /$ day for five days) was started for the treatment of clinical suspected of GBS. After the treatment, the facial movements improved, dysphagia slowly resolved, and limb numbness disappeared. The deep tendon 
reflexes were present but still hypoactive in the lower limbs. Two weeks later, an elevated titer of IgM anti-GQ1B was reported. Four weeks after the onset of his symptoms, he had no complaints and the results from his neurologic examination were found normal.

\section{Discussion}

At present, the diagnosis of GBS is based on the diagnostic criteria of Asbury and Cornblath [4] and MFS which is a clinical trial of total external ophthalmoplegia, ataxia, and areflexia [5]. Although the symptoms and signs of the case presentation were not clearly separated between GBS and MFS. The most common initial symptoms and signs of anti-GQ1b antibody are also accompanied by antecedent illness in upper respiratory infection, diplopia, gait disturbance and external ophthalmoplegia, however this patient was considered a variant of GBS, because it is reported that patients presented with MFS may progress to GBS [6]. Over 90\% of MFS cases have acute phase anti-GQ1b ganglioside antibody which is particularly associated with ophthalmologic disease such as botulism [7]. MFS has been described as an unusual variant of GBS with a benign prognosis. In overlapping MFS and GBS, there have been reports of symptoms and signs that are rarely found, including dysesthesia, dysarthria, facial weakness, bulbar palsy, decreased deep tendon reflex and sensory dysfunction which are similar to this patient's condition [6].

\section{Conclusion}

Serum anti-GQ1b antibody is not intended to be used as a laboratory diagnosis alone, but recognition of anti-GQ1b antibodyrelated disease is useful for understanding the etiological relation among the various illnesses and for introducing the established treatments with unusual clinical presentations $[8,9]$.

\section{References}

1. Yoshikawa K, Kuwahara M, Morikawa M, Fukumoto Y, Yamana M, et al. (2018) Varied antibody reactivities and clinical relevance in anti-GQ1b antibody-related diseases. Neurol Neuroimmunol \& Neuroinflamm 5(6).

2. Morikawa M, Kuwahara M, Ueno R, Samukawa M, Hamada Y, et al. (2016) Serological study using glycoarray for detecting antibodies to glycolipids and glycolipid complexes in immune-mediated neuropathies. J Neuroimmunol 301: 35-40.

3. Paparounas K (2004) Anti-GQ1b ganglioside antibody in peripheral nervous system disorders pathophysiologic role and clinical relevance. Arch Neurol 61(7): 1013-1016.

4. Asbury AK, Cornblath DR (1990) Assessment of current diagnostic criteria for Guillain Barre syndrome. Ann Neurol 27(suppl): S21-S24.

5. Fisher M (1956) An unusual variant of acute idiopathic polyneuritis (syndrome of ophthalmoplegia, ataxia and areflexia). N Engl J Med 255: 57-65.

6. Odaka M, Yuki N, Hirata K (2001) Anti-GQ1b IgG antibody syndrome: Clinical and immunological range. J Neurol Neurosurg Psychiatry 70(1): 50-55.

7. Boylu EE, Toğrol RE, Senol MG, Ozdağ MF, Saraçoğlu M (2010) Role of anti-GQ1B antibody in differential diagnosis of acute ophthalmoparesis. Neuropsychiatr Dis Treat 6: 119-122.

8. Ogawara K, Kuwabara S, Yuki N (2002) Fisher syndrome or Bickerstaff brainstem encephalitis? anti-GQ1b IgG antibody syndrome involving both the peripheral and central nervous system. Muscle Nerve 26(6): 845-849.

9. Chiba A, Kusunoki S, Shimizu T, Kanazawa I (1992) Serum IgG antibody to ganglioside GQ1b is a possible marker of Miller Fisher syndrome. Ann Neurol 31(6): 677-679.
ISSN: 2574-1241

DOI: 10.26717/BJSTR.2019.13.002334

Sarawut Suksuphew. Biomed J Sci \& Tech Res



This work is licensed under Creative

Commons Attribution 4.0 License

Submission Link: https://biomedres.us/submit-manuscript.php

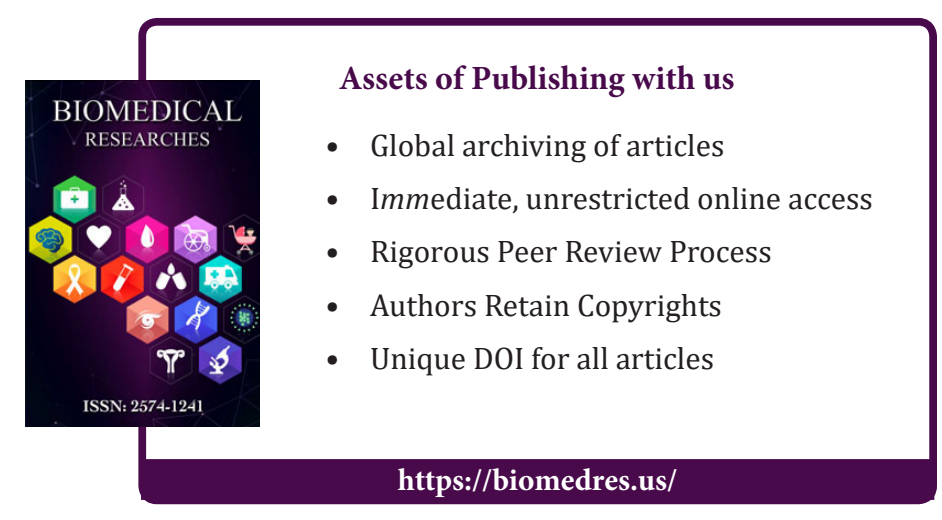

